\section{The phenotype of congenital insensitivity to pain due to the Nav1.9 variant p.L811P}

European Journal of Human Genetics (2015) 23, 561-563; doi:10.1038/ejhg.2014.166; published online 13 August 2014

Individuals with congenital insensitivity to pain (CIP) have never felt pain despite possessing an anatomically normal peripheral nervous system and normal intelligence. ${ }^{1}$ In 2006/7, biallelic null mutations in the transmembrane voltage-gated sodium channel $\mathrm{Na}_{\mathrm{V}} 1.7 /$ SCN9A were discovered as a cause of CIP. ${ }^{2,3}$ Recently, a new form of CIP was reported in two isolated unrelated cases. Both had recurrent injuries and self-mutilations secondary to feeling no pain, and identical de novo heterozygous p.L811P variants in the voltage-gated sodium channel $\mathrm{Na}_{V} 1.9$, encoded by SCN11A. ${ }^{4}$ We have now identified a further case with the identical variant c.2432T $>$ C (p.(L811P)) and report that all have a complex extended phenotype, see Table 1.

The presenting feature in all three was failure to thrive secondary to intestinal dysmotility. Consequent upon this, all had multiple hospital admissions and investigations and two required parenteral nutrition. Abnormal gut peristalsis was found; however, intestinal biopsies were repeatedly normal. All have continuing problems with diarrhoea and/ or constipation.

All three have severe pruritus, scratching themselves sufficiently to cause full-thickness skin loss in the cervical area during infancy (see Figure 1). Remarkably this was at the same location as seen in transgenic Scn1la-mutant mice. ${ }^{4}$ Ulceration and itching may be secondary to hyperhidrosis, as itching only reduced following the use of cyprohepatidine, which lead to a clear reduction in sweating. Hyperhidrosis persisted throughout life, and increased on exertion and raised ambient temperatures. All cried and blushed normally, and none had any abnormal cardiovascular findings nor emotional liability.

Motor milestones were delayed, consequences of a persisting hypotonia and mild muscle weakness. No clinical or significant neurophysiological signs of peripheral neuropathy were observed. Two individuals adopted bizarre dystonia-like postures at the extremes of normal joint positions, awake and asleep.
All have slow wound healing, and patient 3 in particular had Staphylococci repeatedly cultured from skin lesions. This apparent selective reduced immunity to staphylococcal infections is also seen in the hereditary autonomic and sensory neuropathy types 4 and 5, caused by biallelic mutations in NTRK1 and NGF, respectively.

No peripheral pain was felt, for example, painless bone fractures and self-amputation of the tongue tip and lips, however, defecation produced significant discomfort. Furthermore, whereas temperature within the normal range could be perceived, variations in temperature such as a gust of cold wind were distinctly unpleasant. These were the only experiences of 'pain' that the individuals describe.

$\mathrm{Na}_{\mathrm{V}} 1.9$ is strongly expressed in enteric plexus and nociceptor/ temperature sensing neurons, which patient biopsies show are present. Therefore the phenotype is mainly caused by nerve dysfunction and not by its absence. The p.L811P variant causes a complex pattern of effects on neuronal subtype activity: it is excitatory in the enteric plexus $\left(\mathrm{Na}_{\mathrm{V}} 1.9\right.$ knockout mice have increased intestinal activity $^{5}$ ), in sweat glands leading to hyperhidrosis and in ano-rectal nociceptors showing these innervations are functioning; but it is inhibitory in most nociceptors and in infective inflammation (which also occur in sensory neuropathies where small unmyelinated nerves are absent). The Na $1.9-C I P$ phenotype is unique and clearly clinically distinguishable from $\mathrm{Na}_{V} 1.7-\mathrm{CIP}$, which is accompanied by anosmia but not gastrointestinal motility disturbances or muscle weakness. In contrast to these channelopathies, the NTRK1associated hereditary autonomic and sensory neuropathy type 4 (also termed 'congenital insensitivity to pain with anhidrosis') is characterized by variable degree of intellectual disability and lack of sweat gland innervation, resulting in anhidrosis and recurrent febrile episodes owing to poor thermoregulation. The complex pathophysiological basis of the $\mathrm{Na}_{\mathrm{V}} 1.9-\mathrm{CIP}$ is further illustrated by the report of families with a dominant episodic pain syndrome caused by missense SCN11A/Na 1.9 variants and by further missense variants leading to adult onset painful neuropathy. ${ }^{6,7}$ All mutations including p.L811P show gain-offunction properties at a channel level and suggest neuron hyperexcitability. ${ }^{4,6,7}$ Yet the contrary physiological outcome depending on the variant, that is, increased versus abolished pain perception, remains puzzling. One explanation might be the effect size of the respective mutation on $\mathrm{Na}_{\mathrm{V}} 1.9$ function that determines the physiological consequences.

This recent insight in human $\mathrm{Na}_{V} 1.9$ pathology suggests that channel agonists and antagonists could have multiple therapeutic potentials.

Information regarding the SCN11A variant c.2432T >C (p.Leu811Pro) (NM_014139.2) is available: OMIM Mutation ID 604385.0001 http://omim.org/entry/604385\#0001; ClinVar: http://www.ncbi.nlm. nih.gov/clinvar/RCV000074494/

Patient consent had been obtained. 
Table 1 A comparison of the phenotype of each individual with congenital insensitivity to pain due to SCN11A/Nav1.9 variant p.L811P

\begin{tabular}{llll}
\hline Phenotype & Patient 1 & Patient 2 & Patient 3 \\
\hline Origin & German & Swedish Scottish \\
Mutation in SCN11A/Nav1.9 & p.L811P, de novo & p.L811P, de novo p.L811P, de novo
\end{tabular}

Neuro-cutaneous features

Peripheral pain felt

Self-inflicted injuries

Predilection of skin ulcers to cervical region

Slow healing wounds

Intolerance of moderate cold and moderate heat, but can sense temperature

change

Sweats more than expected for ambient temperature or exercise done

Pruritus

Axon flare response

Can produce tears, can blush, but can't be tickled

Gastrointestinal features

Significant problems with failure to thrive secondary to intestinal

dysmotility

Diarrhoea

Continuing problems with constipation

Abdominal discomfort coinciding with constipation

Perineal discomfort on passing motion or urine

Episodic abdominal pain (not coinciding with constipation)

Rectal pain on defecation (similar to paroxysmal extreme pain disorder due

to Nav1.7 mutations)

Intermittent parenteral nutrition

Neurodevelopmental profile

Delayed motor milestones

Intelligence

Emotions, mood, personality

\section{Neurologic features}

Intact sense of smell

Persisting minor hypotonia and muscle weakness

Adopts postures at extremes of joint movement range when awake

(giving an appearance similar to dystonia)

Adopts postures at extremes of joint movement range when asleep

Standard nerve conduction studies

Sural nerve biopsy

\section{Musculoskeletal features \\ Multiple painless fractures \\ Charcot-like arthropathy}

\section{Pharmacologic features}

Response of pain episodes to carbamazepine

(and some other sodium channel blockers, eg, lamotrigine)

Response of pruritus to standard anti-histamines

$\begin{array}{lll}\text { No } & \text { No } & \text { No } \\ \text { Yes } & \text { Yes } & \text { Yes } \\ \text { Yes } & \text { Yes } & \text { Yes } \\ \text { Yes } & \text { Yes } & \text { Yes } \\ \text { Yes } & \text { Yes } & \text { Yes } \\ & & \\ \text { Yes } & \text { Yes } & \text { Yes } \\ \text { Yes } & \text { Yes } & \text { Yes } \\ \text { No } & \text { No } & \text { Yes } \\ \text { Yes } & \text { Yes } & \text { Yes }\end{array}$

$\begin{array}{lll}\text { Yes } & \text { Yes } & \text { Yes }\end{array}$

Yes (episodes of diarrhoea/ Yes (continuous Yes (during first constipation)

Imodium therapy)

1-2 years of life)

$?$

?

No

No

No

Yes

Yes Yes

Yes Yes

No Yes

No Yes $\quad$ Yes

Yes

Yes

No

Yes

Yes

Yes

Normal (born 2002) Normal (born 2004)

Normal (born 2007)

Normal

Normal

Normal

Yes

Yes

Yes

Yes

Yes

Yes

Yes

Yes

Normal

No

Yes

Normal

No

Yes

Borderline

Normal

Not done

Not done

Yes

Yes

Yes

Yes

Yes

Yes

Not done

Not done

No

Not done

No

No 


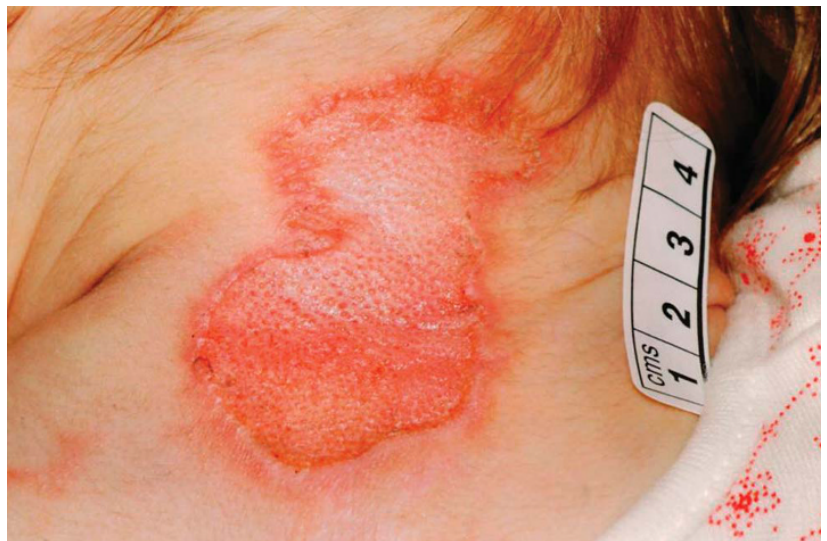

Figure 1 Neck of case 3 with the SCN11A/Nav1.9 mutation p.L811P at the age of 1 year 5 months. A large contiguous healing region is shown. This was caused by the child scratching, mostly while awake. This area was excoriated for 6 months due to intense itching for which hyperhidrosis was a significant contributory factor.

\section{CONFLICT OF INTEREST}

CGW is a Principal Investigator of an MRC MICA grant including Neusentis. The remaining authors declare no conflict of interest.

\section{ACKNOWLEDGEMENTS}

This work is dedicated to the memory of Dr John Tolmie who sadly passed away during its final preparation. John was a highly regarded geneticist as well as a clinician, an inspiring colleague and above all a great person.

Christopher Geoffrey Woods ${ }^{4}$, Mohamed Osman Eltahir Babiker ${ }^{2}$, Iain Horrocks ${ }^{2}$, John Tolmie ${ }^{3}$ and Ingo Kurth ${ }^{\star, 4}$

${ }^{1}$ Department of Medical Genetics, Cambridge Institute for Medical Research, Addenbrooke's Hospital, Cambridge, UK;

${ }^{2}$ Fraser of Allander Neurosciences Unit, Royal Hospital for Sick Children, Glasgow, Scotland, UK;

${ }^{3}$ Department of Clinical Genetics, Southern General Hospital, Glasgow, Scotland, UK;

${ }^{4}$ Institute of Human Genetics, Jena University Hospital, Jena, Germany E-mail: ingo.kurth@med.uni-jena.de

1 Görke W: The differential diagnosis of congenital analgesia and other diseases with diminished pain perception in childhood. Case report and review. Neuropediatrics 1981; 12: 33-44.

2 Cox JJ, Reimann F, Nicholas AK et al: An SCN9A channelopathy causes congenital inability to experience pain. Nature 2006: 444: 894-898.

3 Goldberg YP, MacFarlane J, MacDonald ML et al: Loss-of-function mutations in the Nav1.7 gene underlie congenital indifference to pain in multiple human populations. Clin Genet 2007; 71: 311-319.

4 Leipold E, Liebmann L, Korenke GC et al: A de novo gain-of-function mutation in SCN11A causes loss of pain perception. Nat Genet 2013; 45: 1399-1404.

5 Copel C, Clerc N, Osorio N, Delmas P, Mazet B: The Nav1.9 channel regulates colonic motility in mice. Front Neurosci 2013; 7: 58

6 Huang J, Han C, Estacion M et al: Gain-of-function mutations in sodium channel Nav1.9 in painful neuropathy. Brain 2014; 137(Pt 6): 1627-1642.

7 Zhang XY, Wen J, Yang W et al: Gain-of-function mutations in SCN11A cause familial episodic pain. Am J Hum Genet 2013; 93: 957-966.

\section{Connexin 26 variant carriers have a better gastrointestinal health: is this the heterozygote advantage?}

European Journal of Human Genetics (2015) 23, 563-564; doi:10.1038/ejhg.2014.151; published online 6 August 2014

Connexin 26 (GJB2) is one of the major factors in human deafness worldwide. On average $1 / 1000$ children is born deaf and the most common GJB2 variant (c.35delG) causes $\sim 40 \%$ of cases in Southern Europe. Carriers' frequency for different loss-of-function GJB2 mutations is very high worldwide (up to $3 \%$ ), ${ }^{1}$ suggesting a heterozygous advantage for a global condition or a founder effect. ${ }^{2}$ In this light, epidermal thickening in GJB2 carriers $^{3,4}$ has been proposed as a possible advantage reducing infections and bacterial invasion through skin. ${ }^{5,6}$ Moreover, in vitro functional studies demonstrated that the loss-of-functional GJB2 expression provides improved protection against gastrointestinal bacterial pathogens. ${ }^{7}$ In particular, enteropathogenic Escherichia coli and Shigella flexneri may induce a strong selective effect, being the most common causes of diarrhoea. Thus, GJB2 carriers might have an increased resistance to gastrointestinal infectious diseases, as already proposed by Simpson et al. ${ }^{7}$

To test this hypothesis, a cross-sectional study involving 203 subjects aged 19-65 years (63\% women) was carried out. Subjects (170) were wildtype for the GJB2 gene, whereas 33 carried one or more variants. The information about diarrhoea episodes and frequency, medical history and covariates (sex and age) was collected. People self-reporting diarrhoea episodes at least once a year were set as cases, whereas the remaining ones were controls (all the subject had similar level of education) (Table 1; Figure 1). Subjects affected by pertinent chronic diseases (Crohn's disease, Intestinal bowel disease and so on) were excluded from the study. Fisher's exact test was performed for case/control proportion in relation to genotype, giving a significant result $(P=0.007)$, with an odds ratio $(\mathrm{OR})$ of $3.21(95 \%$ confidence interval (CI): 1.27-9.24). Although our sample was sex and age homogeneous (Wilcoxon test $P>0.05$ ), performing the same analysis separated by sex revealed that females mainly contributed to the finding $(P=0.0016)$. In particular, in our data set women had higher incidence of diarrhoea than men ( $46 \%$ and $36 \%$ respectively), and for female GJB2 carriers this proportion dropped to $12.5 \%$, with an $\mathrm{OR}=8.33(95 \% \mathrm{CI}: 1.82-78.03)$. As regards to the reported frequency per die (range: 0-3), linear regression was applied, 